\section{Insomnia among Patients with Chronic Heart Failure}

\section{Fotos NV*}

Department of Nursing, School of Health Sciences, National and Kapodistrian University of Athens, Greece

Received: June 10, 2018; Accepted: June 12, 2018; Published: June 19, 2018
Heart failure (HF) is a chronic, progressive disease, a complex clinical syndrome that results from any structural or functional disorder of the heart. It is defined as the inability of the heart to function effectively according to metabolic requirements of the body [1]. HF is a common disease, especially among the elderly, and is one of the most important causes of morbidity and mortality worldwide [2]. In United States of America, about 650,000 people are diagnosed with HF every year, with the disease being one of the most common causes of admission to hospital [1,3]. Also, there are 15 million patients with HF in Europe [4].

Clinical manifestations of HF include dyspnea, fatigue, nocturia, and fluid retention that can lead to pulmonary edema, peripheral edema and ascites [4]. Over the past few years has been a global scientific concern for less life-threatening but extremely troublesome manifestations of $\mathrm{HF}$, such as insomnia and its effects on the daily life and quality of life of patients with HF. Insomnia is a persistent sleep disorder, characterized by the difficulty of initiating and/or maintaining sleep, while early morning awakenings is a common problem. As a result, feeling of daytime fatigue, reduced concentration, headache, irritability and psychological disorders are caused [5].

Príncipe-Rodríguez et al. in their study [6] examined patients with HF for sleep disturbance symptoms and correlated symptoms with markers of clinical status. Among participants, $31.3 \%$ reported symptoms of chronic insomnia. Paroxysmal nocturnal dyspnea and New York Heart Association status classification were associated with complaint of insomnia. Broström et al. [7] found that the most common sleep disturbances among patients with HF were difficulty in initiating, maintaining sleep, and early morning awakenings, while $21 \%$ of patients referred daytime sleepiness. The above sleep disorders were associated significantly with poor quality of life.

In the study of Garcia et al. [8], 159 older adult patients with HF examined for their sleep quality and cognitive function. It was found that problems most commonly reported by the patients were difficulties falling asleep (20.8\%), staying asleep (49.6\%) and interruptions in sleep due to bathroom use (56.0\%). Impaired sleep has been associated with poorer cognitive function, reduced quality of life and increased depressive symptomatology.

\author{
*Corresponding author: Fotos NV \\ ”nikfotos@nurs.uoa.gr

\begin{abstract}
Assistant Professor of Medical Nursing, Department of Nursing, School of Health Sciences, National and Kapodistrian University of Athens, 23 Papadiamadopoulou Str, 11527, Goudi, Athens, Greece.
\end{abstract}

Tel: 00302107461454

Citation: Fotos NV (2018) Insomnia among Patients with Chronic Heart Failure. J Hosp Med Manage Vol.4 No.1:6

In another study, Wang et al. [9] found that eight to ten (81\%) patients with HF reported poor sleep quality. Sleep efficacy and duration of sleep during the night were the main problems. The most common reason for sleep interruption was the urination during the night. Also, female patients, those who had selfperceived poor health, comorbidities and depression were related with poor sleep quality. Insomnia was reported by $32 \%$ of patients with HF in a recent study of Chimluang et al. [10]. Most mentioned problems with sleep were difficulty falling asleep, difficulty staying asleep, and waking up too early. Factors correlated with insomnia were depression, anxiety, marital status (divorced, widowed) and dyspnea.

According to abovementioned studies insomnia is common among patients with HF. Other investigators tried to found methods for reducing the frequency and severity of insomnia and diminishing the effects of it on patients with HF. In a randomised controlled trial of Suna et al. [11], the effect of a supervised exercise training programme on sleep quality in recently discharged heart failure patients was examined. Almost half of patients with $\mathrm{HF}$ at baseline reported poor sleep quality. Females and those with severe HF reported insomnia more frequently, while patients with poor sleep quality reported significantly more depression symptoms. Patients participated in exercise training 
programme showed significantly greater improvement in sleep quality. The greater improvement was detected in patients with reduced difficulty breathing at night.

In conclusion, insomnia and poor sleep quality are of major concern for patients with HF. Insomnia is an undervalued problem by healthcare professionals, and has a significant

\section{References}

1. Yancy CW, Jessup M, Bozkurt B, Butler J, Casey DE Jr (2013) 2013 ACCF/ AHA. Guideline for the management of heart failure: A Report of the American College of Cardiology Foundation/American Heart Association Task Force on Practice Guidelines. Circulation 128: 240-327.

2. Go AS, Mozaffarian D, Roger VL, Benjamin EJ, Berry JD, et al. (2013) Heart disease and stroke statistics-2013 update: a report from the American Heart Association. Circulation 127: 6-245.

3. Roger LV (2013) Epidemiology of Heart Failure. Circ Res 113: 646-659.

4. McMurray JJ, Adamopoulos S, Anker SD, Auricchio A, Böhm M (2012) ESC Guidelines for the diagnosis and treatment of acute and chronic heart failure. Eur Heart J 14: 803-869.

5. https://sleepfoundation.org/insomnia/content/what-causesinsomnia

6. Rodríguez PK, Strohl KP, Hadziefendic S, Piña IL (2005) Sleep negative impact on functional capacity, self-care and quality of life of patients with HF. The international scientific community must be more concerned with addressing the problem, aiming at the development of insomnia prevention methods that can be applied in primary health care facilities and by the patients in their home. symptoms and clinical markers of illness in patients with heart failure. Sleep Breath 9: 127-133.

7. Broström A, Strömberg A, Dahlström U, Fridlund B (2004) Sleep difficulties, daytime sleepiness, and health-related quality of life in patients with chronic heart failure. J Cardiovasc Nurs 19: 234-242.

8. Garcia S, Alosco ML, Spitznagel MB, Cohen R, Raz N, et al. (2012) Poor sleep quality and reduced cognitive function in persons with heart failure. Int J Cardiol 156: 248-249.

9. Wang $T$, Lee $S$, Tsay $S$, Tung $H$ (2010) Factors influencing heart failure patients' sleep quality. J Adv Nurs 66: 1730-1740.

10. Chimluang J, Aungsuroch $Y$, Jitpanya C (2017) Descriptors of insomnia among patients with heart failure. J Med Assoc Thai 100: 403-409.

11. Suna JM, Mudge A, Stewart I, Marquart L, O'Rourke P, et al. (2015) The effect of a supervised exercise training programme on sleep quality in recently discharged heart failure patients. Eur J Cardiovasc Nurs 14: 198-205. 